Mathematical Modelling AND ANALysis

Volume 16 Number 4, December 2011, 537-548

http://dx.doi.org/10.3846/13926292.2011.627951

(c) Vilnius Gediminas Technical University, 2011
Publisher: Taylor\&Francis and VGTU

http://www.tandfonline.com/TMMA

Online ISSN: $1648-3510$

Print ISSN: 1392-6292

\title{
The Finite Part of Divergent Integrals with Logarithmic Factors*
}

\section{Kaido Lätt}

Institute of Mathematics, University of Tartu

J.Liivi 2, 50409 Tartu, Estonia

E-mail(corresp.): klatt@ut.ee

Received May 22, 2011; revised August 29, 2011; published online November 1, 2011

\begin{abstract}
The concepts of the finite part (f.p.) and analytic finite part (a.f.p.) of divergent integrals are defined in the situation where the singular function in the integral has a logarithmic factor. The change of variables in f.p.- and a.f.p-integrals is examined.
\end{abstract}

Keywords: divergent integral, finite part, analytic finite part, change of variables.

AMS Subject Classification: 40A10; 32A55.

\section{Introduction}

Divergent integrals and equations containing them have been studied for a long time, including the principal work done by Hadamard in [5]. Equations containing divergent integrals have been useful in applications in mathematics $[1,6,7,10]$ and physics $[8,9]$. One of the concepts under discussion has been the summability, i.e. finding the finite part (f.p.), of divergent integrals. Over the years numerous approaches to defining the finite part of divergent integrals have been examined (see $[5,7]$ ). For methods on numerically finding the finite part of divergent integrals see $[2,3,4]$. In [11], a unified approach to summability of divergent integrals is presented that covers the approaches considered before. Later, in [12] summability methods for divergent integrals are studied in more detail in the case where the integrand is represented as a product of two functions, one with a parameter-dependent non-integrable singularity at one point of integration and the other absolutely integrable. Under discussion are methods which are based on the expansion of the absolutely integrable function in a Taylor series with center at the singular point (f.p.) and on the analytic continuation with respect to the parameter of the singularity (a.f.p.). Formulae of changes of variables in such integrals are also presented.

* This work was supported by the Estonian Science Foundation grant 7353 . 
More precisely, in [12], divergent integrals

$$
\int_{0}^{R} a(r) r^{-\lambda-1} d r
$$

depending on $\lambda \in \mathbb{C}$, are examined under assumptions

$$
a \in C^{m, \alpha}[0, R], \quad m \in \mathbb{N}_{0}=\{0,1,2, \ldots\}, \quad 0<\alpha \leqslant 1,
$$

where $C^{m, \alpha}[0, R]$ is the class of functions satisfying the conditions $a \in C^{m}[0, R]$, $\left|a^{(m)}(r)-a^{(m)}(0)\right| \leqslant M r^{\alpha}, 0 \leqslant r \leqslant R ; M$ is a positive constant.

For $\lambda \in \mathbb{C}$ with $\operatorname{Re} \lambda<m+\alpha$ the finite part of (1.1) is defined in terms of Taylor expansions: if $\lambda \in \mathbb{C} \backslash \mathbb{N}_{0}$, then

$$
\begin{aligned}
\text { f.p. } \int_{0}^{R} a(r) r^{-\lambda-1} d r= & \int_{0}^{R}\left[a(r)-\sum_{k=0}^{m} \frac{1}{k !} a^{(k)}(0) r^{k}\right] r^{-\lambda-1} d r \\
& +\sum_{k=0}^{m} \frac{1}{k !} a^{(k)}(0) \frac{R^{k-\lambda}}{k-\lambda} ;
\end{aligned}
$$

while if $\lambda=l \in \mathbb{N}_{0}$, then

$$
\begin{aligned}
\text { f.p. } \int_{0}^{R} a(r) r^{-l-1} d r= & \int_{0}^{R}\left[a(r)-\sum_{k=0}^{m} \frac{1}{k !} a^{(k)}(0) r^{k}\right] r^{-l-1} d r \\
& +\sum_{\substack{k=0 \\
k \neq l}}^{m} \frac{1}{k !} a^{(k)}(0) \frac{R^{k-l}}{k-l}+\frac{1}{l !} a^{(l)}(0) \ln R .
\end{aligned}
$$

These definitions have two crucial consequences. Firstly, the finite part integral defined by (1.3) is the analytic continuation of integral (1.1) from $\operatorname{Re} \lambda<0$ into $\left\{\lambda \in \mathbb{C} \backslash \mathbb{N}_{0} \mid \operatorname{Re} \lambda<m+\alpha\right\}$. Secondly, for $\lambda=l \in \mathbb{N}_{0}$

$$
\text { f.p. } \int_{0}^{R} a(r) r^{-l-1} d r=\lim _{\substack{\lambda \rightarrow l \\ \lambda \notin \mathbb{N}_{0}}} \frac{d}{d \lambda}\left[(\lambda-l) \text { f.p. } \int_{0}^{R} a(r) r^{-\lambda-1} d r\right] \text {. }
$$

For the change of variables $r=g(\rho)$ with

$$
g \in C^{m+1, \alpha}\left[0, R_{*}\right], \quad g(0)=0, \quad g\left(R_{*}\right)=R, \quad g^{\prime}(\rho)>0, \quad 0 \leqslant \rho \leqslant R_{*}
$$

the following result is established in [12].

Theorem 1. Assume that (1.2) and (1.4) hold. Then for $\operatorname{Re} \lambda<m+\alpha$,

$$
\text { f.p. } \int_{0}^{R} a(r) r^{-\lambda-1} d r=\text { f.p. } \int_{0}^{R_{*}} a_{*}(\rho, \lambda) \rho^{-\lambda-1} d \rho+\Pi_{*}(\lambda),
$$

where $a_{*}(\rho, \lambda)=a(g(\rho))(g(\rho) / \rho)^{-\lambda-1} g^{\prime}(\rho)$ and

$$
\Pi_{*}(\lambda)= \begin{cases}0, & \lambda \in \mathbb{C} \backslash \mathbb{N}_{0}, \\ -\left.\frac{1}{l !} \frac{\partial}{\partial \lambda}\left(\frac{\partial}{\partial \rho}\right)^{l} a_{*}(\rho, \lambda)\right|_{\rho=0, \lambda=l}, & \lambda=l \in \mathbb{N}_{0} .\end{cases}
$$


The analytic finite part of integrals $\int_{0}^{R} a(r, \lambda) r^{-\lambda-1} d r$ is also defined. Here $a(r, \lambda)$, with regards to $\lambda \in \mathbb{C}$, is an analytic function. Moreover, the formula for change of variables in a.f.p.-integrals is given:

$$
\text { a.f.p. } \int_{0}^{R} a(r, \lambda) r^{-\lambda-1} d r=\text { a.f.p. } \int_{0}^{R_{*}} a_{*}(\rho, \lambda) \rho^{-\lambda-1} d \rho
$$

with $a_{*}(\rho, \lambda)=a(g(\rho), \lambda)(g(\rho) / \rho)^{-\lambda-1} g^{\prime}(\rho)$.

The first goal of the present paper is to define the finite part of integral

$$
\int_{0}^{R} a(r) r^{-\lambda-1}(\ln r)^{n} d r
$$

with $n \in \mathbb{N}_{0}$ so that the f.p.-integrals have the same two crucial properties they had in [12]. We will also show that a similar result to Theorem 1 holds for change of variables in the f.p.-integrals of (1.5). In the last part of the present article we define the analytic finite part of integral $\int_{0}^{R} a(r, \lambda) r^{-\lambda-1}(\ln r)^{n} d r$ and examine the change of variables in a.f.p.-integrals. As it turns out, the logarithmic factor $(\ln r)^{n}$ greatly complicates the situation. For instance, the correction term $\Pi_{*}(\lambda)$ in the formula for the change of variables now contains $n+1$ terms (see Theorem 2).

\section{The Finite Part of a Divergent Integral}

We consider integrals (1.5) where $\lambda \in \mathbb{C}, n \in \mathbb{N}_{0}$,

$$
a \in C^{m, \alpha}[0, R], \quad m \in \mathbb{N}_{0}, \quad 0<\alpha \leqslant 1
$$

and $C^{m, \alpha}[0, R]$ is the class of functions that satisfy conditions

$$
a \in C^{m}[0, R], \quad\left|a^{(m)}(r)-a^{(m)}(0)\right| \leqslant M r^{\alpha}, \quad 0 \leqslant r \leqslant R ;
$$

$M>0$ is a constant. For $\operatorname{Re} \lambda<0$ integral (1.5) converges; for $\operatorname{Re} \lambda \geqslant 0$ it generally diverges.

Let $\lambda \in \mathbb{C}$, $\operatorname{Re} \lambda<m+\alpha$. We define the finite part of (1.5) in the following way: for $\lambda \in \mathbb{C} \backslash \mathbb{N}_{0}$

$$
\begin{aligned}
& \text { f.p. } \int_{0}^{R} a(r) r^{-\lambda-1}(\ln r)^{n} d r=\int_{0}^{R}\left[a(r)-\sum_{k=0}^{m} \frac{1}{k !} a^{(k)}(0) r^{k}\right] r^{-\lambda-1}(\ln r)^{n} d r \\
& \quad+\sum_{k=0}^{m} \frac{1}{k !} a^{(k)}(0) R^{k-\lambda} \sum_{j=0}^{n}(-1)^{n-j} \frac{n !}{j !} \frac{(\ln R)^{j}}{(k-\lambda)^{n-j+1}}
\end{aligned}
$$

while for $\lambda=l \in \mathbb{N}_{0}$

$$
\begin{aligned}
& \text { f.p. } \int_{0}^{R} a(r) r^{-l-1}(\ln r)^{n} d r=\int_{0}^{R}\left[a(r)-\sum_{k=0}^{m} \frac{1}{k !} a^{(k)}(0) r^{k}\right] r^{-l-1}(\ln r)^{n} d r \\
& +\sum_{\substack{k=0 \\
k \neq l}}^{m} \frac{1}{k !} a^{(k)}(0) R^{k-l} \sum_{j=0}^{n}(-1)^{n-j} \frac{n !}{j !} \frac{(\ln R)^{j}}{(k-l)^{n-j+1}}+\frac{1}{l !} a^{(l)}(0) \frac{(\ln R)^{n+1}}{n+1} .
\end{aligned}
$$


Both definitions (2.3) and (2.4) are based on the expansion of the absolutely integrable function $a$ in a Taylor series with centre at the singular point (cf. [11]). To demonstrate that the integrals on the right-hand sides of (2.3) and (2.4) converge absolutely for $\operatorname{Re} \lambda<m+\alpha$, we show, similarly to the way it was done in [12], that (2.2) leads to

$$
\left|a(r)-\sum_{k=0}^{m} \frac{1}{k !} a^{(k)}(0) r^{k}\right| \leqslant \frac{M}{m !} r^{m+\alpha}, \quad 0 \leqslant r \leqslant R .
$$

If $m=0$, then (2.5) is obvious. For $m \geqslant 1$, by Taylor's formula with integral remainder term we have

$$
\begin{aligned}
a(r) & -\sum_{k=0}^{m-1} \frac{1}{k !} a^{(k)}(0) r^{k}=\frac{1}{(m-1) !} \int_{0}^{r}(r-\rho)^{m-1} a^{(m)}(\rho) d \rho \\
= & \frac{1}{(m-1) !} \int_{0}^{r}(r-\rho)^{m-1}\left[a^{(m)}(\rho)-a^{(m)}(0)\right] d \rho+\frac{1}{m !} a^{(m)}(0) r^{m},
\end{aligned}
$$

that is,

$$
a(r)-\sum_{k=0}^{m} \frac{1}{k !} a^{(k)}(0) r^{k}=\frac{1}{(m-1) !} \int_{0}^{r}(r-\rho)^{m-1}\left[a^{(m)}(\rho)-a^{(m)}(0)\right] d \rho .
$$

Now (2.5) follows from (2.2) and (2.6).

As mentioned before, one of the goals of this article was to define the finite part of integral (1.5) so that f.p.-integrals would have the same two crucial properties they had in article [12]. To be more precise, for $\lambda \in \mathbb{C} \backslash \mathbb{N}_{0}$, the f.p.-integral defined by (2.3) should be the analytic continuation of (1.5), and for points $\lambda=l \in \mathbb{N}_{0}$, a certain limit relation should hold. We close out this section by showing that with (2.3) and (2.4) we have achieved our goals.

Firstly, the f.p.-integral defined by $(2.3)$ is the analytic continuation of (1.5) from $\operatorname{Re} \lambda<0$ into $D=\left\{\lambda \in \mathbb{C} \backslash \mathbb{N}_{0} \mid \operatorname{Re} \lambda<m+\alpha\right\}$ because the right-hand side of (2.3) is an analytic function of $\lambda \in \mathbb{C}$ in the domain $D$ and for $\operatorname{Re} \lambda<0$ (cf. $(2.3))$

$$
\text { f.p. } \int_{0}^{R} a(r) r^{-\lambda-1}(\ln r)^{n} d r=\int_{0}^{R} a(r) r^{-\lambda-1}(\ln r)^{n} d r .
$$

Secondly, for integer points $\lambda=l \in \mathbb{N}_{0}$

$$
\begin{aligned}
\text { f.p. } & \int_{0}^{R} a(r) r^{-l-1}(\ln r)^{n} d r \\
= & \lim _{\substack{\lambda \rightarrow l \\
\lambda \notin \mathbb{N}_{0}}}\left(\frac{d}{d \lambda}\right)^{n+1}\left[\frac{(\lambda-l)^{n+1}}{(n+1) !} \text { f.p. } \int_{0}^{R} a(r) r^{-\lambda-1}(\ln r)^{n} d r\right] .
\end{aligned}
$$

To show (2.7) we use the fact that for a function $f$ analytic at $\lambda=l$, it holds that

$$
\lim _{\lambda \rightarrow l}\left(\frac{d}{d \lambda}\right)^{n+1}\left[\frac{(\lambda-l)^{n+1}}{(n+1) !} f(\lambda)\right]=\lim _{\lambda \rightarrow l} f(\lambda)=f(l)
$$


Thus, by definition (2.3),

$$
\begin{aligned}
\lim _{\substack{\lambda \rightarrow l \\
\lambda \notin \mathbb{N}_{0}}}\left(\frac{d}{d \lambda}\right)^{n+1}\left[\frac{(\lambda-l)^{n+1}}{(n+1) !} \mathrm{f} . \mathrm{p} . \int_{0}^{R} a(r) r^{-\lambda-1}(\ln r)^{n} d r\right] \\
=\int_{0}^{R}\left[a(r)-\sum_{k=0}^{m} \frac{1}{k !} a^{(k)}(0) r^{k}\right] r^{-l-1}(\ln r)^{n} d r \\
\quad+\sum_{\substack{k=0 \\
k \neq l}}^{m} \frac{1}{k !} a^{(k)}(0) R^{k-l} \sum_{j=0}^{n}(-1)^{n-j} \frac{n !}{j !} \frac{(\ln R)^{j}}{(k-l)^{n-j+1}}+\Psi(l),
\end{aligned}
$$

with

$$
\begin{aligned}
\Psi(l)= & -\frac{1}{l !} a^{(l)}(0) \lim _{\substack{\lambda \rightarrow l \\
\lambda \notin \mathbb{N}_{0}}} \sum_{j=0}^{n} \frac{1}{j !(n+1)}(\ln R)^{j}\left(\frac{d}{d \lambda}\right)^{n+1}\left[(\lambda-l)^{j} R^{l-\lambda}\right] \\
= & -\frac{1}{l !} a^{(l)}(0) \lim _{\substack{\lambda \rightarrow l \\
\lambda \notin \mathbb{N}_{0}}} \sum_{j=0}^{n} \sum_{u=0}^{n+1} \frac{j !}{j !(n+1)(j-u) !}\left(\begin{array}{c}
n+1 \\
u
\end{array}\right) \\
& \times(\lambda-l)^{j-u}(-1)^{n+1-u}(\ln R)^{n+1-u+j} R^{l-\lambda} .
\end{aligned}
$$

Since terms with $u \neq j$ converge to zero as $\lambda \rightarrow l$, we get

$$
\begin{aligned}
\Psi(l)=- & \frac{1}{l !} \frac{1}{n+1} a^{(l)}(0)(\ln R)^{n+1}(-1)^{n+1} \lim _{\substack{\lambda \rightarrow l \\
\lambda \notin \mathbb{N}_{0}}} R^{l-\lambda} \\
\times & {\left[\sum_{j=0}^{n+1}\left(\begin{array}{c}
n+1 \\
j
\end{array}\right)(-1)^{j}-\left(\begin{array}{c}
n+1 \\
n+1
\end{array}\right)(-1)^{n+1}\right] . }
\end{aligned}
$$

Bearing in mind that $\sum_{j=0}^{n+1}\left(\begin{array}{c}n+1 \\ j\end{array}\right)(-1)^{j}=(1-1)^{n+1}=0$, we obtain $\Psi(l)=$ $\frac{1}{l !} a^{(l)}(0)(\ln R)^{n+1} /(n+1)$. Hence, (2.7) holds. Moreover, the proof of $(2.7)$ shows us that

$$
\begin{aligned}
& \text { f.p. } \int_{0}^{R} a(r) r^{-l-1}(\ln r)^{i} d r \\
& =\lim _{\substack{\lambda \rightarrow l \\
\lambda \notin \mathbb{N}_{0}}}\left(\frac{d}{d \lambda}\right)^{n+1}\left[\frac{(\lambda-l)^{n+1}}{(n+1) !} \text { f.p. } \int_{0}^{R} a(r) r^{-\lambda-1}(\ln r)^{i} d r\right]
\end{aligned}
$$

holds for $i=0,1, \ldots, n$.

\section{The Change of Variables in f.p.-Integrals}

Before we consider the change of variables in f.p.-integrals, notice that f.p.integrals defined by (2.3) and (2.4) for different $\lambda \in \mathbb{C}$ are independent of one another if $a \in C^{m, \alpha}[0, R]$. It means one can apply these definitions also in the case of functions $a(r, \lambda)$ which are dependent of $\lambda \in \mathbb{C}$ as a parameter. For 
example we use $\left.\left(\frac{\partial}{\partial r}\right) a(r, \lambda)\right|_{r=0}$ in place of $a^{(k)}(0)$ on the right-hand side of (2.3) when defining $\int_{0}^{R} a(r, \lambda) r^{-\lambda-1}(\ln r)^{n} d r, \lambda \in \mathbb{C} \backslash \mathbb{N}_{0}$.

We now discuss the concept of change of variables $r=g(\rho)$ in f.p.-integrals (2.3) and (2.4), where

$$
g \in C^{m+1, \alpha}\left[0, R_{*}\right], \quad g(0)=0, \quad g\left(R_{*}\right)=R, \quad g^{\prime}(\rho)>0, \quad 0 \leqslant \rho \leqslant R_{*} .
$$

One can easily see that $(3.1)$ implies $g(\rho)=\rho g_{1}(\rho)$ with $g_{1} \in C^{m, \alpha}\left[0, R_{*}\right]$ and $g_{1}(\rho)>0$ for all $\rho \in\left[0, R_{*}\right]$.

Theorem 2. Assume that (2.1) and (3.1) hold. Then for $\operatorname{Re} \lambda<m+\alpha$,

$$
\text { f.p. } \int_{0}^{R} a(r) r^{-\lambda-1}(\ln r)^{n} d r=\sum_{i=0}^{n}\left(\begin{array}{c}
n \\
i
\end{array}\right) \text { f.p. } \int_{0}^{R_{*}} a_{i}(\rho, \lambda) \rho^{-\lambda-1}(\ln \rho)^{i} d \rho+\Pi_{*}(\lambda),
$$

where $a_{i}(\rho, \lambda)=a(g(\rho)) g_{1}(\rho)^{-\lambda-1} g^{\prime}(\rho)\left(\ln g_{1}(\rho)\right)^{n-i}, i=0,1, \ldots, n$, and

$$
\Pi_{*}(\lambda)= \begin{cases}0, & \lambda \in \mathbb{C} \backslash \mathbb{N}_{0}, \\
-\left.\sum_{i=0}^{n}\left(\begin{array}{c}
n \\
i
\end{array}\right) \frac{1}{l !} \frac{1}{i+1}\left(\frac{\partial}{\partial \lambda}\right)^{i+1}\left(\frac{\partial}{\partial \rho}\right)^{l} a_{i}(\rho, \lambda)\right|_{\rho=0, \lambda=l}, & \lambda=l \in \mathbb{N}_{0} .\end{cases}
$$

Proof. Observe that under our assumptions about $a$ and $g$, functions $a_{i}(\rho, \lambda)$, $i=0,1, \ldots, n$, belong to $C^{m, \alpha}\left[0, R_{*}\right]$. Therefore, according to the remark we made at the beginning of this section, the f.p.-integrals on the right-hand side of (3.2) are correctly defined.

Every f.p.-integral in (3.2), as a function of $\lambda$, is analytic in the domain $\left\{\lambda \in \mathbb{C} \backslash \mathbb{N}_{0} \mid \operatorname{Re} \lambda<m+\alpha\right\}$. For $\operatorname{Re} \lambda<0$, integral (1.5) is absolutely convergent and therefore we can make a change of variables $r=g(\rho)$ :

$$
\int_{0}^{R} a(r) r^{-\lambda-1}(\ln r)^{n} d r=\sum_{i=0}^{n}\left(\begin{array}{c}
n \\
i
\end{array}\right) \int_{0}^{R_{*}} a_{i}(\rho, \lambda) \rho^{-\lambda-1}(\ln \rho)^{i} d \rho .
$$

Hence, (3.2) holds for $\lambda \in \mathbb{C} \backslash \mathbb{N}_{0}$ with $\Pi_{*}(\lambda)=0$.

Now let $\lambda=l \in \mathbb{N}_{0}, l<m+\alpha$. In such case we have (2.7), on the righthand side of which, for points $\lambda \in \mathbb{C} \backslash \mathbb{N}_{0}$, one can make a change of variables according to $(3.2)$ :

$$
\begin{aligned}
\text { f.p. } & \int_{0}^{R} a(r) r^{-l-1}(\ln r)^{n} d r=\lim _{\substack{\lambda \rightarrow l \\
\lambda \notin \mathbb{N}_{0}}}\left(\frac{d}{d \lambda}\right)^{n+1}\left[\frac{(\lambda-l)^{n+1}}{(n+1) !} \mathrm{f} . \mathrm{p} \cdot \int_{0}^{R} a(r) r^{-\lambda-1}(\ln r)^{n} d r\right] \\
= & \sum_{i=0}^{n}\left(\begin{array}{c}
n \\
i
\end{array}\right) \lim _{\substack{\lambda \rightarrow l \\
\lambda \notin \mathbb{N}_{0}}}\left(\frac{d}{d \lambda}\right)^{n+1}\left[\frac{(\lambda-l)^{n+1}}{(n+1) !} \mathrm{f} \cdot \mathrm{p} \cdot \int_{0}^{R_{*}} a_{i}(\rho, \lambda) \rho^{-\lambda-1}(\ln \rho)^{i} d \rho\right] \\
= & \sum_{i=0}^{n}\left(\begin{array}{c}
n \\
i
\end{array}\right) \lim _{\substack{\lambda \rightarrow l \\
\lambda \notin \mathbb{N}_{0}}}\left(\frac{d}{d \lambda}\right)^{n+1}\left[\frac{(\lambda-l)^{n+1}}{(n+1) !} \mathrm{f} \cdot \mathrm{p} \cdot \int_{0}^{R_{*}} a_{i}(\rho, l) \rho^{-\lambda-1}(\ln \rho)^{i} d \rho\right] \\
& +\sum_{i=0}^{n}\left(\begin{array}{c}
n \\
i
\end{array}\right) \lim _{\substack{\lambda \rightarrow l \\
\lambda \notin \mathbb{N}_{0}}}\left(\frac{d}{d \lambda}\right)^{n+1}\left[\frac{(\lambda-l)^{n+1}}{(n+1) !} \mathrm{f} \cdot \mathrm{p} \cdot \int_{0}^{R_{*}} a_{i}^{*}(\rho, \lambda) \rho^{-\lambda-1}(\ln \rho)^{i} d \rho\right]
\end{aligned}
$$


where $a_{i}^{*}(\rho, \lambda)=a_{i}(\rho, \lambda)-a_{i}(\rho, l)$. Note that $\left(\frac{\partial}{\partial \lambda}\right)^{l} a_{i}^{*}(\rho, \lambda)=\left(\frac{\partial}{\partial \lambda}\right)^{l} a_{i}(\rho, \lambda)$ for $l \in \mathbb{N}$. Keeping in mind the remark we made at the beginning of the present section, (2.9) leads us to

$$
\begin{aligned}
& \lim _{\substack{\lambda \rightarrow l \\
\lambda \notin \mathbb{N}_{0}}}\left(\frac{d}{d \lambda}\right)^{n+1}\left[\frac{(\lambda-l)^{n+1}}{(n+1) !} \text { f.p. } \int_{0}^{R_{*}} a_{i}(\rho, l) \rho^{-\lambda-1}(\ln \rho)^{i} d \rho\right] \\
& \quad=\text { f.p. } \int_{0}^{R_{*}} a_{i}(\rho, l) \rho^{-l-1}(\ln \rho)^{i} d \rho
\end{aligned}
$$

for $i=0,1, \ldots, n$. Therefore we arrive at a formula in the form of (3.2) with

$$
\Pi_{*}(l)=\sum_{i=0}^{n}\left(\begin{array}{c}
n \\
i
\end{array}\right) \lim _{\substack{\lambda \rightarrow l \\
\lambda \notin \mathbb{N}_{0}}}\left(\frac{d}{d \lambda}\right)^{n+1}\left[\frac{(\lambda-l)^{n+1}}{(n+1) !} \text { f.p. } \int_{0}^{R_{*}} a_{i}^{*}(\rho, \lambda) \rho^{-\lambda-1}(\ln \rho)^{i} d \rho\right],
$$

which by definition (2.3) is transformed to

$$
\begin{aligned}
\Pi_{*}(l)= & \sum_{i=0}^{n}\left(\begin{array}{c}
n \\
i
\end{array}\right) \lim _{\substack{\lambda \rightarrow l \\
\lambda \notin \mathbb{N}_{0}}}\left(\frac{d}{d \lambda}\right)^{n+1}\left\{\frac { ( \lambda - l ) ^ { n + 1 } } { ( n + 1 ) ! } \int _ { 0 } ^ { R _ { * } } \left[a_{i}^{*}(\rho, \lambda)\right.\right. \\
& \left.-\left.\sum_{k=0}^{m} \frac{1}{k !}\left(\frac{\partial}{\partial \rho}\right)^{k} a_{i}^{*}(\rho, \lambda)\right|_{\rho=0} \rho^{k}\right] \rho^{-\lambda-1}(\ln \rho)^{i} d \rho+\frac{(\lambda-l)^{n+1}}{(n+1) !} \\
& \left.\times\left.\sum_{k=0}^{m} \frac{1}{k !}\left(\frac{\partial}{\partial \rho}\right)^{k} a_{i}^{*}(\rho, \lambda)\right|_{\rho=0} R_{*}^{k-\lambda} \sum_{j=0}^{i}(-1)^{i-j} \frac{i !}{j !} \frac{\left(\ln R_{*}\right)^{j}}{(k-\lambda)^{i-j+1}}\right\} .
\end{aligned}
$$

The integrand approaches zero as $\lambda \rightarrow l$ for each $\rho \in\left[0, R_{*}\right]$ and is dominated by a function $c \rho^{m+\alpha-\lambda-1}(\ln \rho)^{i+n+1}$, which is integrable for $\operatorname{Re} \lambda<m+\alpha$ (cf. (2.5)). Hence, by Lebesgue's theorem the integral also converges to zero as $\lambda \rightarrow l$. In conclusion, in a neighbourhood of $\lambda=l$ the integral and terms in the sum with $k \neq l$ are analytic functions (cf. (2.8)) and converge to zero as $\lambda \rightarrow l$ and thus

$$
\begin{aligned}
\Pi_{*}(l)= & \sum_{i=0}^{n}\left(\begin{array}{c}
n \\
i
\end{array}\right) \frac{1}{l !} \lim _{\substack{\lambda \rightarrow l \\
\lambda \notin \mathbb{N}_{0}}}\left(\frac{d}{d \lambda}\right)^{n+1}\left[\left.\frac{(\lambda-l)^{n+1}}{(n+1) !}\left(\frac{\partial}{\partial \rho}\right)^{l} a_{i}^{*}(\rho, \lambda)\right|_{\rho=0} R_{*}^{l-\lambda}\right. \\
& \left.\times \sum_{j=0}^{i}(-1)^{i-j} \frac{i !}{j !} \frac{\left(\ln R_{*}\right)^{j}}{(l-\lambda)^{i-j+1}}\right] \\
= & -\sum_{i=0}^{n}\left(\begin{array}{c}
n \\
i
\end{array}\right) \frac{1}{l !} \lim _{\substack{\lambda \notin l \\
\lambda \notin \mathbb{N}_{0}}} \sum_{j=0}^{i}\left(\ln R_{*}\right)^{j} \frac{i !}{(n+1) ! j !}\left(\frac{d}{d \lambda}\right)^{n+1}\left[(\lambda-l)^{n-i+j}\right. \\
& \left.\times\left.\left(\frac{\partial}{\partial \rho}\right)^{l} a_{i}^{*}(\rho, \lambda)\right|_{\rho=0} R_{*}^{l-\lambda}\right] \\
= & -\sum_{i=0}^{n}\left(\begin{array}{c}
n \\
i
\end{array}\right) \frac{1}{l !} \lim _{\substack{\lambda \rightarrow l \\
\lambda \notin \mathbb{N}_{0}}} \sum_{j=0}^{i} \sum_{u=0}^{n+1}\left(\begin{array}{c}
n+1 \\
u
\end{array}\right) \frac{i !(n-i+j) !}{(n+1) ! j !(n-i+j-u) !} \\
& \times(\lambda-l)^{n-i+j-u}\left(\ln R_{*}\right)^{j}\left(\frac{d}{d \lambda}\right)^{n+1-u}\left[\left.\left(\frac{\partial}{\partial \rho}\right)^{l} a_{i}^{*}(\rho, \lambda)\right|_{\rho=0} R_{*}^{l-\lambda}\right] .
\end{aligned}
$$


Since the terms in the sum with $u \neq n-i+j$ converge to zero as $\lambda \rightarrow l$,

$$
\begin{aligned}
\Pi_{*}(l)= & -\sum_{i=0}^{n}\left(\begin{array}{c}
n \\
i
\end{array}\right) \frac{1}{l !} \lim _{\substack{\lambda \rightarrow l \\
\lambda \notin \mathbb{N}_{0}}} \sum_{j=0}^{i} \sum_{v=0}^{i-j+1}\left(\begin{array}{c}
n+1 \\
n-i+j
\end{array}\right)\left(\begin{array}{c}
i-j+1 \\
v
\end{array}\right) \frac{i !(n-i+j) !}{(n+1) ! j !} \\
& \times\left(\frac{\partial}{\partial \lambda}\right)^{v}\left[\left.\left(\frac{\partial}{\partial \rho}\right)^{l} a_{i}^{*}(\rho, \lambda)\right|_{\rho=0}\right](-1)^{i-j+1-v}\left(\ln R_{*}\right)^{i+1-v} R_{*}^{l-\lambda} \\
= & -\sum_{i=0}^{n}\left(\begin{array}{c}
n \\
i
\end{array}\right) \frac{1}{l !} \lim _{\substack{\lambda \notin l \\
\lambda \notin \mathbb{N}_{0}}} \sum_{v=1}^{i+1} R_{*}^{l-\lambda}\left(\frac{\partial}{\partial \lambda}\right)^{v}\left[\left.\left(\frac{\partial}{\partial \rho}\right)^{l} a_{i}^{*}(\rho, \lambda)\right|_{\rho=0}\right]\left(\ln R_{*}\right)^{i+1-v} \\
& \times \sum_{j=0}^{i+1-v}\left(\begin{array}{c}
n+1 \\
n-i+j
\end{array}\right)\left(\begin{array}{c}
i-j+1 \\
v
\end{array}\right) \frac{i !(n-i+j) !}{(n+1) ! j !}(-1)^{i-j+1-v} .
\end{aligned}
$$

The last equality holds because $\lim _{\lambda \rightarrow l, \lambda \notin \mathbb{N}_{0}} a_{i}^{*}(\rho, \lambda)=0$. As $\sum_{j=0}^{r}\left(\begin{array}{l}r \\ j\end{array}\right)(-1)^{j}=$ $(1-1)^{r}=0$ for $r \neq 0$, then

$$
\begin{aligned}
\Pi_{*}(l)= & -\sum_{i=0}^{n}\left(\begin{array}{c}
n \\
i
\end{array}\right) \frac{1}{l !} \frac{1}{i+1} \lim _{\substack{\lambda \rightarrow l \\
\lambda \notin \mathbb{N}_{0}}} \sum_{v=1}^{i+1} R_{*}^{l-\lambda}\left(\frac{\partial}{\partial \lambda}\right)^{v}\left[\left.\left(\frac{\partial}{\partial \rho}\right)^{l} a_{i}(\rho, \lambda)\right|_{\rho=0}\right] \\
& \times\left(\ln R_{*}\right)^{i+1-v}(-1)^{i+1-v}\left(\begin{array}{c}
i+1 \\
v
\end{array}\right) \sum_{j=0}^{i+1-v}\left(\begin{array}{c}
i+1-v \\
j
\end{array}\right)(-1)^{j} \\
= & -\left.\sum_{i=0}^{n}\left(\begin{array}{c}
n \\
i
\end{array}\right) \frac{1}{l !} \frac{1}{i+1}\left(\frac{\partial}{\partial \lambda}\right)^{i+1}\left(\frac{\partial}{\partial \rho}\right)^{l} a_{i}(\rho, \lambda)\right|_{\rho=0, \lambda=l} .
\end{aligned}
$$

Thus, (3.2) holds also for $\lambda=l \in \mathbb{N}_{0}$.

\section{The Analytic Finite Part of a Divergent Integral}

Let us now examine integrals

$$
\int_{0}^{R} a(r, \lambda) r^{-\lambda-1}(\ln r)^{n} d r,
$$

where $n \in \mathbb{N}_{0}$ and $a(r, \lambda)$ satisfies the following conditions:

(AN1) for fixed $r \in[0, R]$ the coefficient $a(r, \lambda)$, as a function of $\lambda$, is analytic;

(AN2) for fixed $\lambda \in \mathbb{C}$ the coefficient $a(r, \lambda)$, as a function of $r$ belongs to $C^{m, \alpha}[0, R]$, and $\left|\left(\frac{\partial}{\partial r}\right)^{m} a(r, \lambda)-\left(\frac{\partial}{\partial r}\right)^{m} a(r, \lambda)\right|_{r=0} \mid \leqslant M_{\lambda} r^{\alpha}, 0 \leqslant r \leqslant R$ with an $M_{\lambda}$ that is bounded on bounded subsets of $\mathbb{C}$.

We define the analytic finite part of divergent integral (4.1) as follows: for $\lambda \in \mathbb{C} \backslash \mathbb{N}_{0}, \operatorname{Re} \lambda<m+\alpha$

$$
\begin{aligned}
& \text { a.f.p. } \int_{0}^{R} a(r, \lambda) r^{-\lambda-1}(\ln r)^{n} d r=\text { f.p. } \int_{0}^{R} a(r, \lambda) r^{-\lambda-1}(\ln r)^{n} d r \\
& =\int_{0}^{R}\left[a(r, \lambda)-\left.\sum_{k=0}^{m} \frac{1}{k !}\left(\frac{\partial}{\partial r}\right)^{k} a(r, \lambda)\right|_{r=0} r^{k}\right] r^{-\lambda-1}(\ln r)^{n} d r
\end{aligned}
$$




$$
+\left.\sum_{k=0}^{m} \frac{1}{k !}\left(\frac{\partial}{\partial r}\right)^{k} a(r, \lambda)\right|_{r=0} R^{k-\lambda} \sum_{j=0}^{n}(-1)^{n-j} \frac{n !}{j !} \frac{(\ln R)^{j}}{(k-\lambda)^{n-j+1}} ;
$$

while for $\lambda=l \in \mathbb{N}_{0}, l<m+\alpha$

$$
\begin{aligned}
\text { a.f.p. } & \int_{0}^{R} a(r, l) r^{-l-1}(\ln r)^{n} d r \\
= & \lim _{\substack{\lambda \rightarrow l \\
\lambda \notin \mathbb{N}_{0}}}\left(\frac{\partial}{\partial \lambda}\right)^{n+1}\left[\frac{(\lambda-l)^{n+1}}{(n+1) !} \text { a.f.p. } \int_{0}^{R} a(r, \lambda) r^{-\lambda-1}(\ln r)^{n} d r\right] .
\end{aligned}
$$

Before we study the change of variables in a.f.p.-integrals we show that

$$
\begin{aligned}
& \text { a.f.p. } \int_{0}^{R} a(r, l) r^{-l-1}(\ln r)^{n} d r=\text { f.p. } \int_{0}^{R} a(r, l) r^{-l-1}(\ln r)^{n} d r \\
& -\left.\frac{1}{l !} \frac{1}{n+1}\left(\frac{\partial}{\partial \lambda}\right)^{n+1}\left(\frac{\partial}{\partial r}\right)^{l} a(r, \lambda)\right|_{r=0, \lambda=l}
\end{aligned}
$$

with $l \in \mathbb{N}_{0}, l<m+\alpha$. To demonstrate the last equality we use formula (4.2) for points $\lambda \in \mathbb{C} \backslash \mathbb{N}_{0}$ on the right-hand side of (4.3) and note that by (AN1) the integral and the terms in the corresponding sum with $k \neq l$ are analytic functions at $\lambda=l$. Thus (cf. (2.8))

$$
\begin{array}{ll}
\text { a.f.p. } & \int_{0}^{R} a(r, l) r^{-l-1}(\ln r)^{n} d r \\
= & \int_{0}^{R}\left[a(r, l)-\left.\sum_{k=0}^{m} \frac{1}{k !}\left(\frac{\partial}{\partial r}\right)^{k} a(r, l)\right|_{r=0} r^{k}\right] r^{-l-1}(\ln r)^{n} d r \\
& +\left.\sum_{\substack{k=0 \\
k \neq l}}^{m} \frac{1}{k !}\left(\frac{\partial}{\partial r}\right)^{k} a(r, l)\right|_{r=0} R^{k-l} \sum_{j=0}^{n}(-1)^{n-j} \frac{n !}{j !} \frac{(\ln R)^{j}}{(k-l)^{n-j+1}}+\Phi(l)
\end{array}
$$

with

$$
\begin{aligned}
\Phi(l)= & \lim _{\substack{\lambda \rightarrow l \\
\lambda \notin \mathbb{N}_{0}}}\left(\frac{\partial}{\partial \lambda}\right)^{n+1}\left[\left.\frac{(\lambda-l)^{n+1}}{(n+1) !} \frac{1}{l !}\left(\frac{\partial}{\partial r}\right)^{l} a(r, \lambda)\right|_{r=0} R^{l-\lambda}\right. \\
& \left.\times \sum_{j=0}^{n}(-1)^{n-j} \frac{n !}{j !} \frac{(\ln R)^{j}}{(l-\lambda)^{n-j+1}}\right] \\
= & -\frac{1}{l !} \frac{1}{n+1} \lim _{\substack{\lambda \rightarrow l \\
\lambda \notin \mathbb{N}_{0}}} \sum_{j=0}^{n} \frac{1}{j !}(\ln R)^{j}\left(\frac{\partial}{\partial \lambda}\right)^{n+1}\left[\left.(\lambda-l)^{j}\left(\frac{\partial}{\partial r}\right)^{l} a(r, \lambda)\right|_{r=0} R^{l-\lambda}\right] .
\end{aligned}
$$

As terms with $j \neq u$ converge to zero as $\lambda \rightarrow l$, we have

$$
\begin{aligned}
\Phi(l)= & -\frac{1}{l !} \frac{1}{n+1} \lim _{\substack{\lambda \rightarrow l \\
\lambda \notin \mathbb{N}_{0}}} \sum_{j=0}^{n} \sum_{u=0}^{n+1}\left(\begin{array}{c}
n+1 \\
u
\end{array}\right) \frac{j !}{(j-u) ! j !}(\ln R)^{j} \\
& \times(\lambda-l)^{j-u}\left(\frac{\partial}{\partial \lambda}\right)^{n+1-u}\left[\left.\left(\frac{\partial}{\partial r}\right)^{l} a(r, \lambda)\right|_{r=0} R^{l-\lambda}\right]
\end{aligned}
$$




$$
\begin{aligned}
= & -\frac{1}{l !} \frac{1}{n+1} \lim _{\substack{\lambda \rightarrow l \\
\lambda \notin \mathbb{N}_{0}}} \sum_{j=0}^{n} \sum_{v=0}^{n+1-j}\left(\begin{array}{c}
n+1 \\
j
\end{array}\right)\left(\begin{array}{c}
n+1-j \\
v
\end{array}\right)(-1)^{n+1-j-v} \\
& \times\left.\left(\frac{\partial}{\partial \lambda}\right)^{v}\left(\frac{\partial}{\partial r}\right)^{l} a(r, \lambda)\right|_{r=0}(\ln R)^{n+1-v} R^{l-\lambda} .
\end{aligned}
$$

Since $\sum_{j=0}^{r}\left(\begin{array}{c}r \\ j\end{array}\right)(-1)^{j}=0$ for $r \neq 0$,

$$
\begin{aligned}
\Phi(l)= & -\left.\frac{1}{l !} \frac{1}{n+1} \lim _{\substack{\lambda \notin l \\
\lambda \notin \mathbb{N}_{0}}} \sum_{v=1}^{n+1} R^{l-\lambda}\left(\frac{\partial}{\partial \lambda}\right)^{v}\left(\frac{\partial}{\partial r}\right)^{l} a(r, \lambda)\right|_{r=0}(\ln R)^{n+1-v} \\
& \times(-1)^{n+1-v}\left(\begin{array}{c}
n+1 \\
v
\end{array}\right) \sum_{j=0}^{n+1-v}\left(\begin{array}{c}
n+1-v \\
j
\end{array}\right)(-1)^{j} \\
& -\frac{1}{l !} \frac{1}{n+1} \lim _{\substack{\lambda \notin l \\
\lambda \notin \mathbb{N}_{0}}}\left\{\left.\left(\frac{\partial}{\partial r}\right)^{l} a(r, \lambda)\right|_{r=0}(\ln R)^{n+1} R^{l-\lambda}(-1)^{n+1}\right. \\
& \left.\times\left[\sum_{j=0}^{n+1}\left(\begin{array}{c}
n+1 \\
j
\end{array}\right)(-1)^{j}-(-1)^{n+1}\left(\begin{array}{c}
n+1 \\
n+1
\end{array}\right)\right]\right\} \\
= & \frac{1}{l !} \\
& \left.\left(\frac{\partial}{\partial r}\right)^{l} a(r, l)\right|_{r=0} \frac{(\ln R)^{n+1}}{n+1}-\left.\frac{1}{l !} \frac{1}{n+1}\left(\frac{\partial}{\partial \lambda}\right)^{n+1}\left(\frac{\partial}{\partial r}\right)^{l} a(r, \lambda)\right|_{r=0, \lambda=l}
\end{aligned}
$$

Hence, according to (2.4) formula (4.4) holds.

To conclude, we show that, as in article [12], the biggest advantage of a.f.p. concept over f.p. is that the formula for change of variables does not contain a correction term. That is, the following result holds for change of variables in a.f.p.-integrals.

Theorem 3. Assume that $a(r, \lambda)$ satisfies conditions (AN1) and (AN2) and $g(\rho)$ satisfies conditions (3.1). Then for $\operatorname{Re} \lambda<m+\alpha$, both in the case of $\lambda \in \mathbb{C} \backslash \mathbb{N}_{0}$ and the case of $\lambda=l \in \mathbb{N}_{0}$, one has the formula

$$
\text { a.f.p. } \int_{0}^{R} a(r, \lambda) r^{-\lambda-1}(\ln r)^{n} d r=\sum_{i=0}^{n}\left(\begin{array}{c}
n \\
i
\end{array}\right) \text { a.f.p. } \int_{0}^{R_{*}} a_{i}(\rho, \lambda) \rho^{-\lambda-1}(\ln \rho)^{i} d \rho
$$

with $a_{i}(\rho, \lambda)=a(g(\rho), \lambda) g_{1}(\rho)^{-\lambda-1} g^{\prime}(\rho)\left(\ln g_{1}(\rho)\right)^{n-i}, i=0,1, \ldots, n$.

Proof. If $\lambda \in \mathbb{C} \backslash \mathbb{N}_{0}, \operatorname{Re} \lambda<m+\alpha$, then according to (4.2) a.f.p. is equal to f.p. and thus (4.5) follows from Theorem 2 .

Now let $\lambda=l \in \mathbb{N}_{0}, l<m+\alpha$. The analytic finite part of integral (4.1) is defined by (4.3), on the right-hand side of which, for points $\lambda \in \mathbb{C} \backslash \mathbb{N}_{0}$, we can make the change of variables

$$
\text { a.f.p. } \int_{0}^{R} a(r, \lambda) r^{-\lambda-1}(\ln r)^{n} d r=\text { f.p. } \int_{0}^{R} a(r, \lambda) r^{-\lambda-1}(\ln r)^{n} d r
$$




$$
=\sum_{i=0}^{n}\left(\begin{array}{l}
n \\
i
\end{array}\right) \text { f.p. } \int_{0}^{R_{*}} a_{i}(\rho, \lambda) \rho^{-\lambda-1}(\ln \rho)^{i} d \rho
$$

with $a_{i}(\rho, \lambda)=a(g(\rho), \lambda) g_{1}(\rho)^{-\lambda-1} g^{\prime}(\rho)\left(\ln g_{1}(\rho)\right)^{n-i}, i=0,1, \ldots, n$. So,

$$
\begin{aligned}
& \text { a.f.p. } \int_{0}^{R} a(r, l) r^{-l-1}(\ln r)^{n} d r=\sum_{i=0}^{n}\left(\begin{array}{c}
n \\
i
\end{array}\right) \lim _{\substack{\lambda \rightarrow l \\
\lambda \notin \mathbb{N}_{0}}}\left(\frac{\partial}{\partial \lambda}\right)^{n+1} \\
& \times\left[\frac{(\lambda-l)^{n+1}}{(n+1) !} \text { f.p. } \int_{0}^{R_{*}} a_{i}(\rho, \lambda) \rho^{-\lambda-1}(\ln \rho)^{i} d \rho\right] .
\end{aligned}
$$

As a consequence of (4.2) and (4.3), formula

$$
\begin{aligned}
& \lim _{\substack{\lambda \rightarrow l \\
\lambda \notin \mathbb{N}_{0}}}\left(\frac{\partial}{\partial \lambda}\right)^{n+1}\left[\frac{(\lambda-l)^{n+1}}{(n+1) !} \text { f.p. } \int_{0}^{R_{*}} a_{i}(\rho, \lambda) \rho^{-\lambda-1}(\ln \rho)^{i} d \rho\right] \\
& \quad=\text { a.f.p. } \int_{0}^{R_{*}} a_{i}(\rho, l) \rho^{-l-1}(\ln \rho)^{i} d \rho
\end{aligned}
$$

holds for $i=0,1, \ldots, n$. By combining (4.6) and (4.7) we obtain (4.5) for $\lambda=l \in \mathbb{N}_{0}$ :

$$
\begin{aligned}
& \text { a.f.p. } \int_{0}^{R} a(r, l) r^{-l-1}(\ln r)^{n} d r \\
& =\sum_{i=0}^{n}\left(\begin{array}{c}
n \\
i
\end{array}\right) \lim _{\substack{\lambda \rightarrow l \\
\lambda \notin \mathbb{N}_{0}}}\left(\frac{\partial}{\partial \lambda}\right)^{n+1}\left[\frac{(\lambda-l)^{n+1}}{(n+1) !} \text { f.p. } \int_{0}^{R_{*}} a_{i}(\rho, \lambda) \rho^{-\lambda-1}(\ln \rho)^{i} d \rho\right] \\
& =\sum_{i=0}^{n}\left(\begin{array}{c}
n \\
i
\end{array}\right) \text { a.f.p. } \int_{0}^{R_{*}} a_{i}(\rho, l) \rho^{-l-1}(\ln \rho)^{i} d \rho .
\end{aligned}
$$

\section{Acknowledgments}

The author would like to thank Gennadi Vainikko and Arvet Pedas for valuable discussion and remarks regarding the manuscript.

\section{References}

[1] S. Belotserkovskiǔ and I. Lifanov. Numerical Methods in Singular Integral Equations and Their Application in Aerodynamics, Elasticity Theory and Electrodynamics. Nauka, Moscow, 1985. (in Russian)

[2] I. Boukov. Passive and Adaptive Algorithms for the Approximate Computation of Singular Integrals. Part 1, 2. Izdatelstvo Penzenskogo Gosudarstvennogo Tekhnicheskogo Universiteta, Penza, 1995. (in Russian)

[3] I. Boukov. Approximate Methods for Computing Singular and Hypersingular Integrals. II. Hypersingular Integrals. Penzenskiı̆ Gosudartsvennyı̆ Universitet, Penza, 2009. (in Russian) 
[4] Yu. Gandel. An Introduction to Numerical Methods for Computing Singular and Hyper-Singular Integrals. Kharkovski Natsionalnii Universitet, Kharkov, 2000. (in Russian)

[5] J. Hadamard. Le probleme de Cauchy et les equations aux derivees partielles lineaires hyperboliques. Hermann, Paris, 1932.

[6] I. Lifanov. The Method of Singular Integral Equations and a Numerical Experiment in Mathematical Physics, Aerodynamics and the Theory of Elasticity and Wave Diffraction. TOO "Yanus", Moscow, 1995. (in Russian)

[7] I. Lifanov. Singular Integral Equations and Discrete Vortices. VSP, Utrecht, 1996.

[8] I. Lifanov, L. Poltavskii and G. Vainikko. Hypersingular Integral Equations and Their Applications, volume 4 of Differ. Integral Equ. Appl. Chapman \& Hall/CRC, Boca Raton, FL, 2004.

[9] G. Monegato. Definitions, properties and applications of finite-part integrals. $J$. Comput. Appl. Math., 229(2):425-439, 2009. http://dx.doi.org/10.1016/j.cam.2008.04.006.

[10] J. Saranen and G. Vainikko. Periodic Integral and Pseudodifferential Equations with Numerical Approximation. Springer Monogr. Math. Springer-Verlag, Berlin, 2002.

[11] G. Vainikko and I. Lifanov. On the notion of the finite part of divergent integrals in integral equations. Differ. Equ., 38(9):1313-1326, 2002. http://dx.doi.org/10.1023/A:1021700706820.

[12] G. Vainikko and I. Lifanov. Approaches to the summability of divergent multidimensional integrals. Sb. Math., 194(7-8):1137-1166, 2003. http://dx.doi.org/10.1070/SM2003v194n08ABEH000760. 